\title{
Discussion on evaporation
}

\section{DISCUSSION OF PENMAN'S INTRODUCTION}

Comments Rider : I should like to make some remarks on Dr. Penman's adress while the equations are still on the board. Dr. Penman has given values of the ratios of the $K$ 's which he has taken from some observations I made a year or two ago. Now the individual values of the $K$ 's from which these ratios are obtained are notoriously difficult to measure with accuracy. For example, any one value of $K_{m}$ is dependent, among other things, on a single observation of $\tau_{o}$ and I have found from experience that the value observed may be in error by about $\pm 15 \%$. The number of observations grouped in the ratios quoted is not large so that the values should be treated with some caution.

Dr. Penman quoted some remarks by Deacon concerning the variation of $\tau$ with height and he was rather perturbed by the statement that for periods of up to 10 minutes DeAcon found considerably variation of $\tau$ with height.

Recently Dr. Robinson (of Kew Observatory) and I have made sircultaneous observations of $\tau_{0}$ by drag plate and $\tau_{z}\left(=-\varrho \overline{u^{\prime} w^{\prime}}\right.$ in the usual notation) by hot wire anemometry at a height of $125 \mathrm{~cm}$. We find that there is reasonable agreement between the $\tau^{\prime} s$ found for periods of about 5 minutes or more.

Dr. Penman said that he felt that the failure of Sverdrup's equation to give agreement with the observed evaporation at Lake Hefner was due to errors in the determination of the lake surface temperature and hence of $q_{0}$. Now the same surface temperature values are used to compute $e_{0}$ for substitution in Surros's equation which was found to fit the observations very well. Perhaps Dr. Penman would care to clarify this point.

Finally it was pointed out, quite correctly, that the Thornthwarte and Holtzmans equation was only strictly applicable when neutral conditions of stability prevailed. We have found at Cambridge that, provided the apparatus used to determine the humidities at the two heights is sited in such a way that the systematic errors due to the stability effect are kept as small as possible, the errors in the determined values of $d$, the zero displacement of the surface, often introduce more uncertainty in the computed value of $E$ over a short period than the neglect of this effect. This is particularly so for a surface such as a tall cereal crop where the value of $d$ varies with wind speed as well as crop height. The failure of this equation to give good agreement with observation at Lake Hefner is explained by the authors of the report on that project. The measurements of temperature and humidity were not determined with sufficient precision.

Reply Penman: I agree that if $q_{0}$ in Sverdrup's equation is too big, so contributing to the badness of fit, then $e_{0}$ in Sutron's equation is too big. It is possible that the goodness of fit is a result of compensating errors, because some of the assumptions in Sutros's equations were not realized during the the experiment.

Comments Monteith : Would Mr. Rrder tell us the conditions of stability to which the ratios of eddy coefficients refer? I believe the observations showed an increase of the ratios with increasing instability. Since days of maximum evaporation are generally days of considerable instability, mean ratios or ratios 
for neutral conditions only obscure the magnitude of the error we may be making when we assume identity of two of the coefficients in order to calculate evaporation.

Reply RIDER: The ratios quoted are for the whole range of stability experienced in the observations.

Comments Dey : I should like to ask what might be the best value of the constant of von Kármán. Is it $0.38,0.40$ or 0.45 as this makes a rather great difference in the value of the evaporation.

Reply RIDER: I use the value of 0.40 for the constant. There have been some determinations in the open, two of which I remember as 0.37 (Pasqulle $1950{ }^{1}$ )) and 0.41 (RIDER $1954^{2}$ )). If an incorrect value is used the computed evaporation will be in error, but the error introduced by deficiencies in the observations of temperature etc. and the neglect of the stability effect are likely to be larger.

Comments Stanill : 1 What agreement is there between calculated and measured values of $H$ in the Heat Balance equation?

2 If the time of bright sunshine varies during the day the values of $H$ will alter considerably.

Reply Penman: I know of no direct measurements of $H$ to show the accuracy of the expression $H=R_{1}(1-r)-R_{B}$. Several experimentators are now using special radiometers for this purpose, and I hope we shall get the kind of check Mr. STANHILl wants in the near future.

The empirical relation between $R_{1}$ and $n / N$ depends on a statistically random distribution of sunshine during the day. For this reason I dislike using it for periods shorter than a week, and prefer a working period of a month. Successful use for a single day must be a happy accident.

Comments DE Vries : a) I want to point to a difficulty in applying SverDRUP's equation $q_{0}$ refers to humidity at the water surface, whereas $u_{0}=0$ is at height $z_{0}$. Since there is a laminar boundary layer between $z=0$ and $z=z_{0}, q_{0}$ may differ appreciably from $q$ at $z=z_{0}$.

b) Although I agree with Dr. Penman that $\tau=\tau_{0}$ in the lowest layers, things may be complicated by the fact that the eqs. refer to stationary conditions, whereas all quantities are functions of the time.

c) Elaboration of experimental data at Wageningen, where simultaneous measurements of solar radiation intensity and duration of sunshine were made during 15 years have shown that the standard deviations of the daily totals computed from a linear regression equation are of the order of 20 to $30 \%$ of the average value.

Comments Businger: Businger draws attention to an improved calculation of evaporation which is set forth in his communication.

1) Pasquill, Proc. Roy. Soc. A. 202 (1950) 143.

2) Rider, Phil. Trans. Roy. Soc. A. 246 (1954) 481. 